\title{
On Spectral Relaxation Method for Entropy Generation on a MHD Flow and Heat Transfer of a Maxwell Fluid
}

\author{
S. Shateyi ${ }^{1 \dagger}$, S. S. Motsa ${ }^{2}$ and Z. Makukula ${ }^{2}$ \\ ${ }^{1}$ University of Venda, Department of Mathematics, Private Bag X5050, Thohoyandou 0950, South Africa \\ ${ }^{2}$ School of Mathematics, Statistics and Computer Science, University of KwaZulu-Natal, \\ Private Bag X01, Scottsville 3209, Pietermaritzburg, South Africa \\ $\dagger$ Corresponding Author Email:stanford.shateyi@univen.ac.za
}

(Received June 8, 2013; accepted January 1, 2014)

\begin{abstract}
The present study investigates entropy generation on a magnetohydrodynamic flow and heat transfer of a Maxwell fluid using a spectral relaxation method. The method is based on simple iteration schemes formed by reduction of the order of the momentum equation followed by a rearrangement of the resulting governing nonlinear equation systems which are then solved using spectral methods. The velocity and temperature profiles are obtained numerically and used to generate the entropy generation number. Entropy generation increased with the Reynolds number, the magnetic parameter and the dimensionless group parameter while decreased for higher Prandtl numbers. The effect of the flow parameters on the velocity and temperature of the flow were also investigated. The results were validated using the $b v p 4 c$ where the spectral relaxation method was found to be accurate and rapidly convergent to the numerical results.
\end{abstract}

Keywords: Spectral relaxation method, Chebyshev collocation, MHD flow, Maxwell fluid.

\section{NOMENCLATURE}

0 uniform magnetic field strength

$\mathrm{Br}$ Brinkman number

$C_{p}$ specific heat at constant temperature

De Deborah number

$u \quad$ velocity component in the $\mathrm{x}$ direction

$v \quad$ velocity component in the y direction

$K$ permeability of the porous medium

$Q \quad$ rate of internal heat generation

$M \quad$ magnetic parameter

$N_{S}$ entropy generation rate

$N_{g}$ local volumetric entropy generation rate

$T$ fluid temperature

$f \quad$ dimensionless stream function

$\mathrm{Pr}$ Prandtl number

Ec Eckert number

$\mathrm{Ha}$ Hartmann number

\section{INTRODUCTION}

The study of non-Newtonian fluid flows over a stretching sheet find applications in numerous manufacturing processes. These include wire and fibre coating, extrusion of molten polymers through a slit die for the production of plastic sheets, and foodstuff

\author{
0 uniform magnetic field strength \\ $\mathrm{Br}$ Brinkman number \\ $c_{p}$ specific heat at constant temperature \\ De Deborah number \\ $u \quad$ velocity component in the $\mathrm{x}$ direction \\ $v \quad$ velocity component in the y direction \\ $K$ permeability of the porous medium \\ $Q \quad$ rate of internal heat generation \\ $M$ magnetic parameter \\ $N_{S}$ entropy generation rate \\ $N_{g}$ local volumetric entropy generation rate \\ $T$ fluid temperature \\ $f$ dimensionless stream function \\ $\mathrm{Pr}$ Prandtl number \\ Ec Eckert number \\ $\mathrm{Ha}$ Hartmann number
}

processing amongst others. In a characteristic sheet production process, the extrudate starts

to solidify as soon as it exits from the die. The quality of the final product depends on the rate of heat transfer and hence the need for the cooling process to be controlled effectively (Mamaloukas et al. 2011; Motsa et al. 2012; Mukhopadhyay 2012). Flows in porous 
channels/tubes have received special interest as they find a number of applications in mechanical engineering and biomedical Hayat et al. (2011). Such flows are applicable in the blood dialysis in artificial kidney, flow in the capillaries, flow in blood oxygenators, the design of filters and design of porous pipe. Hydromagnetics has been a subject of interest lately because of its applications in geophysics and astrophysics. To purify the molten metals from nonmetallic inclusions, hydromagnetic techniques are used hence the significance of studies of magnetohydrodynamic (MHD) flows of electrically conducting fluids (Abel et al. 2012; Mukhopadhyay and Gorla 2012).

Amed and Kalita (2013) analyzed the problem of nonlinear MHD flow with heat and mass transfer characteristics of an incompressible, viscous, electrically conducting and Newtonian fluid over a vertical oscillating porous plate embedded in a porous medium in presence of homogeneous chemical reaction of first order and thermal radiation effects. Kumar (2013) recently reported the effects of radiation and heat source over a stretching surface subjected to a power law heat flux, in the presence of transverse magnetic field on two-dimensional boundary layer steady flow and heat transfer of a viscous incompressible fluid. Muthucumaraswamy et al. (2013) presented an exact analysis of rotation effects on unsteady flow of an incompressible and electrically conducting fluid past a uniformly accelerated infinite isothermal vertical plate with variable mass diffusion, under the action of transversely applied magnetic field.

Non-Newtonian fluids are mainly classified into three types namely differential, rate and integral Hayat et al (2012). The Maxwell model is the simplest subclass of the rate type fluids. This fluid model has become the most popular as it can predict stress relaxation and also excludes the complicating effects of shear-dependent viscosity Mukhopadhyay 2012. In view of the Maxwell model, several researchers have analyzed the MHD flow of a Maxwell fluid under various aspects of rotation, thermal radiation, heat and mass transfer, chemical reaction, suction/injection, thermophoresis and heat source/sink (Bataller 2011; Zheng et al. 2013; Noor 2012; Vajravelu 2012).

According to the second law of thermodynamics, all flow and heat transfer processes undergo changes that are irreversible mostly caused by the energy losses during the processes (Butt and Ali 2013). Such effects cannot be completely eliminated from the system and this results in loss of energy. In thermodynamics, such irreversibility is quantified by means of entropy generation rate (Butt and Ali 2013). The accurate estimation of entropy generation is crucial in the design and development of thermo-fluid components such as pipe networks, energy storage systems, heat exchangers, pumps, turbines, and electronic cooling devices, Mahian et al (2012).

Since the pioneering work carried out by (Bejan 1979, 1982) extensive research has been made by researchers related to the entropy generation in the flow and heat transfer systems. Rashidi and Mehr (2012) investigated the entropy generation in the MHD flow over a porous rotating disk in the presence of the velocity slip and temperature jump conditions by means of the DTM-Padé. From their simulations they found out that the disk surface was a strong source of irreversibility and that entropy was minimized when the magnetic interaction, Prandtl number, Brinkman number decrease or when the Reynolds number and suction parameter increase.

Effects of the magnetic field on the entropy generation on the flow and heat transfer of a viscous fluid due to the radially stretching sheet were considered by Butt and Ali (2013). They carried out their simulations using the homotopy analysis method and the shooting method and found that the local entropy generation number increased with the magnetic field parameter and that the fluid friction and the magnetic field irreversibility were dominant at the stretching surface. Makinde and Gbolagade (2005) analyzed the combined effects of viscous dissipation and inclined channel uniform temperature on the entropy generation and irreversibility ratio on a laminar flow of a viscous incompressible fluid through an inclined channel with isothermal walls. The problem was solved analytically by means of separation of variables. From their results the heat transfer irreversibility showed to dominate along the channel centerline

Karamallah et al. (2011) carried out a numerical study of entropy generation in a vertical square channel packed with saturated porous media. The results reviled that as Darcy and Reynolds numbers were increasing, the irreversibility due to fluid friction dominated, while as the Eckert increased, the irreversibility due to heat transfer increased. The effect of uncertainties in physical properties on entropy generation between two rotating cylinders with nanofluids was investigated by Mahian et al. (2012). They found analytical expressions of the entropy generation number for six different models. From their study it was shown that when the contribution of viscous effects in entropy generation were adequately high for the base fluid, all the models predicted the increase of entropy generation with an increase of particle loading.

Makinde and Aziz (2011) analytically analyzed entropy generation and thermal stability in a long hollow cylinder with asymmetry convective cooling. The internal heat generation irreversibility was found to dominate almost the entire slab. The effect of porous medium permeability on the entropy generation and irreversibility ratio in a laminar flow through a channel filled with saturated porous media was investigated by Makinde and Osalusi (2005). The results showed that heat transfer irreversibility dominated over fluid friction irreversibility and that viscous dissipation had no effect on the entropy generation rate at the centerline of the channel.

A numerical study of entropy generation of a magnetohydrodynamic (MHD) flow and heat transfer over a nonlinear permeable stretching sheet with partial slip at constant surface temperature was presented by Yazdi et al. (2011). The entropy generation number was enhanced by the suction parameter and decreased with the injection parameter because of the friction irreversibility due to viscous dissipations. Also, the 
Brinkman and Reynolds number tended to enhance entropy generation. Eegunjobi and Makinde (2012) carried out a numerical study of the combined effect of buoyancy force and Navier slip on entropy generation in a vertical porous channel. Buoyancy forces caused a slight increase in the entropy generation rate at the injection wall and sporadic increases at the suction wall and as the slip parameter increased, the entropy generation rate at both suction and injection walls increased.

In addition to the above mentioned studies more work has been carried out on entropy generation to consider viscosity and convective cooling (Tshehla et al. 2010; Tshehla and Makinde 2011; Torabi and Aziz 2012), channel inlet port height Shuja et al. 2008, heat transfer and flow in nanofluid suspensions Hassan et al. (2013), thermal radiation Chen et al. (2011) and hydrodynamic slip Butt et al. (2012), amongst others.

The above studies have focused on entropy generation analysis on non-Newtonian fluids and to the best of our knowledge none has been reported on the Maxwell fluid model. In this study we aim at investigating entropy generation on a magnetohydrodynamic flow and heat transfer of a Maxwell fluid over a stretching sheet in a Darcian porous medium. The velocity and temperature profiles will be obtained numerically and used to generate the entropy generation number. The effects of physical flow parameters on the entropy generation number will be investigated. The study will be carried out using the spectral relaxation method. It is a newly proposed numerical scheme used to solve nonlinear systems of boundary value problems. It is based on simple iteration schemes formed by reduction of the order of the momentum equation followed by a rearrangement of the resulting governing nonlinear equation systems which are then solved using the Chebyshev spectral collocation method Motsa and Makukula (2013) and Shateyi and Makinde (2013). In the paper, in section 2 we give the model formulation of the problem, in section 3 the spectral relaxation method is given in detail, in section 4 are the results and discussions and section 5 are the conclusions based on the findings.

\section{Problemformulation}

We consider a steady MHD flow of a Maxwell fluid over stretching sheet in a Darcian porous medium. A uniform magnetic field $B_{0}$ is applied normal to the flow direction and there is no applied electric field. As depicted in Figure 1, the $x$-axis is taken in the direction of the main flow along the plate and the $y-$ axis is normal to the plate with velocity components $u, v$ in these directions. The boundary layer approximations for the flow under consideration are

$$
\frac{\partial u}{\partial x}+\frac{\partial v}{\partial y}=0
$$

$$
\begin{gathered}
u \frac{\partial u}{\partial x}+v \frac{\partial u}{\partial y}+\lambda_{1}\left[u^{2} \frac{\partial^{2} u}{\partial x^{2}}+v^{2} \frac{\partial^{2} u}{\partial y^{2}}+2 u v \frac{\partial^{2} u}{\partial x \partial y}\right] \\
=v \frac{\partial^{2} u}{\partial y^{2}} \\
-\frac{\sigma B_{0}^{2}}{\rho}\left(u+\lambda_{1} v \frac{\partial u}{\partial y}\right) \frac{v}{\kappa} u, \quad(2) \\
u \frac{\partial T}{\partial x}+v \frac{\partial T}{\partial y}-\frac{\sigma B_{0}^{2}}{\rho C_{p}} u^{2} \\
=\frac{\kappa}{\rho C_{p}} \frac{\partial^{2} T}{\partial y^{2}}+\frac{\mu}{\rho C_{p}}\left(\frac{\partial u}{\partial y}\right)^{2} \\
+\frac{Q}{\rho C_{p}}\left(T-T_{\infty}\right) .
\end{gathered}
$$

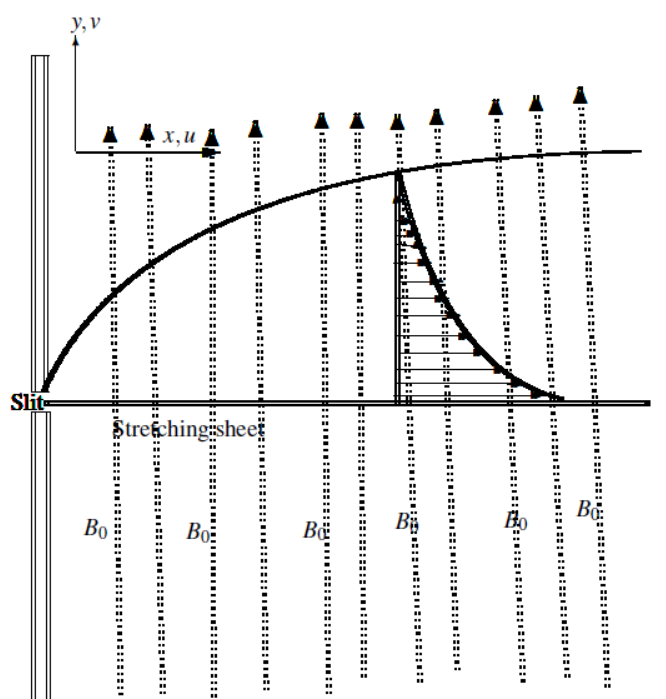

Fig. 1. Physical configuration and coordinate system for the flow

The suitable boundary conditions are

$$
\begin{aligned}
& u=u_{0}(x)=\lambda x, v=0, T=T_{w}(x)=\frac{A x^{2}}{l^{2}}+ \\
& T_{\infty} \quad \text { at } y=0 \text {, } \\
& u \rightarrow 0, \frac{\partial u}{\partial y} \rightarrow 0, T \rightarrow T_{\infty} \text {, as } y \rightarrow \infty \text {, }
\end{aligned}
$$

\subsection{Similarity transformation}

The governing equations (2) - (5) can be transformed to a set of nonlinear ordinary differential equations by introducing the following non-dimensional variables:

$\eta=\sqrt{\frac{\lambda}{v}} y, \psi=\sqrt{\lambda v} x f(\eta), \theta(\eta)=\frac{T-T_{\infty}}{T_{w}-T_{\infty}}$,

where $\psi$ is the stream function that satisfies the continuity equation (1) with

$$
u=\frac{\partial \psi}{\partial y}=\lambda x f^{\prime}(\eta), v=-\frac{\partial \psi}{\partial x}=\sqrt{\lambda v} f(\eta)
$$

Using equations (6) and (7) in the governing equations we obtain the following set of non-linear ordinary differential equations, 


$$
\begin{gathered}
f^{\prime \prime \prime}+(1+M D e) f f^{\prime \prime}-\left(f^{\prime}\right)^{2}+ \\
D e\left(2 f f^{\prime} f^{\prime \prime}-f^{2} f^{\prime \prime \prime}\right)-(\gamma+M) f^{\prime}=0 \\
\frac{1}{\operatorname{Pr}} \theta^{\prime \prime}+f \theta^{\prime}+\left(\Lambda-2 f^{\prime}\right) \theta+E c\left[M\left(f^{\prime}\right)^{2}\right. \\
\left.\left.+f^{\prime \prime}\right)^{2}\right]=0,
\end{gathered}
$$

where $D e=\lambda_{1} \lambda$ is the Deborah number, $M=\frac{\sigma B_{0}^{2}}{\lambda \rho}$ is the magnetic parameter, $\gamma=\frac{v}{\lambda K}$ is the porosity parameter, $\Lambda=\frac{Q}{\lambda \rho c_{p}}$ is the heat source/sink parameter, $\operatorname{Pr}=\frac{\rho c_{p}}{k}$ is the Prandtl number, $E c=$ $\frac{\lambda}{c_{p} A}$ is the Eckert number.

The boundary conditions become:

$f=0, f^{\prime}=1, \theta=1$, at $\eta=0$,

$f^{\prime} \rightarrow 0, \theta \rightarrow 0$, as $\eta \rightarrow \infty$

\subsection{Entropy generation analysis}

We consider the entropy generation related to MHD flow of a Maxwell fluid over a stretching surface. Entropy generation in a fluid is caused by the exchange of momentum and energy within the fluid and at the boundary. Heat transfer in the direction of finite temperature gradients generates one part of the entropy generation while the other part is due to fluid friction irreversibility. The local volumetric rate of entropy generation in the presence of a magnetic field is given by (Woods 1985):

$\mathrm{S}_{\mathrm{g}}=\frac{\kappa}{\mathrm{T}_{\infty}^{2}}\left[\left(\frac{\partial \mathrm{T}}{\partial \mathrm{x}}\right)^{2}+\left(\frac{\partial \mathrm{T}}{\partial \mathrm{y}}\right)^{2}\right]+\frac{\mu}{\mathrm{T}_{\infty}}\left(\frac{\partial \mathrm{u}}{\partial \mathrm{y}}\right)^{2}+\frac{\sigma \mathrm{B}_{0}^{2}}{\mathrm{~T}_{\infty}} \mathrm{u}^{2}$.

The first term on the right-hand side of equation (12) is the entropy generation due to heat transfer across a finite temperature gradient; the second term is the local entropy generation due to viscous dissipation, while the third term is the local entropy generation due to the effect of the magnetic field. We get the dimensionless number for the entropy generation rate $N_{S}$ by dividing the local volumetric entropy generation rate $S_{g}$ by a characteristic entropy rate $S_{g 0}$

For prescribed boundary condition the characteristic entropy generation rate is:

$$
S_{g 0}=\frac{k(\Delta T)^{2}}{l^{2} T_{\infty}^{2}},
$$

where $\Delta T$ is the temperature difference, $l$ is the characteristic length. Therefore, the entropy generation number is

$$
N_{s}=\frac{s_{g}}{s_{g 0}}
$$

Using the expressions of the dimensionless velocity and temperature, the entropy generation number is then given by:

$$
\begin{aligned}
N_{s}=\frac{4}{X^{2}} \theta^{2}+ & R e_{l}\left(\theta^{\prime}\right)^{2} \\
& +\frac{B r}{\Omega}\left(\operatorname{Re}_{l}\left(f^{\prime \prime}\right)^{2}\right. \\
& \left.+H a\left(f^{\prime}\right)^{2}\right),
\end{aligned}
$$

where $R e_{l}=\frac{u_{l} l}{v}$ is Reynolds number based on the characteristic length, $B r=\frac{v u_{0}^{2}}{k \Delta T}$ is the Brinkman number, $H a=B_{0} l \sqrt{\frac{\sigma}{v}}$ is the Hartmann number, $X=x / l$ is the non-dimensional surface length and $\Omega=\frac{\Delta T}{T_{\infty}}$ is the dimensionless temperature difference.

The rate of entropy generation will then be obtained from the previous solutions of the boundary layers for the fluid velocity and temperature.

\section{METHOD of SOLUTION}

In this section we present the development of the spectral relaxation method (SRM) for the solution of the governing equations and its implementation on the governing equations (8 - 9). Before applying the SRM, it is convenient to reduce the order of the momentum equation by introducing the transformation $f^{\prime}(\eta)=g(\eta)$. The transformed equations become

$$
\begin{aligned}
& g^{\prime \prime}+(1+M D e) f g^{\prime}-g^{2}+ \\
& \operatorname{De}\left(2 f g g^{\prime}-f^{2} g^{\prime \prime}\right)-(\gamma+M) g=0, \\
& \quad f^{\prime}=g,(17) \\
& \frac{1}{P r} \theta^{\prime \prime}+f \theta^{\prime}-2 g \theta+\Omega \theta+E c\left[M g^{2}+\left(g^{\prime}\right)^{2}\right]= \\
& 0,
\end{aligned}
$$

and the boundary conditions are

$$
\begin{gathered}
f=0 ; \quad g=1 ; \quad \theta=1 ; \quad \text { at } \eta=0, \\
g \rightarrow 0 ; \quad \theta \rightarrow 0 \quad \text { as } \eta \rightarrow \infty .
\end{gathered}
$$

To develop the SRM algorithm, we consider equations $(16-18)$ in the order in which they are listed and label them as equations for $g, f$ and $\theta$ respectively. The labelling strategy is guided by the highest order derivatives in each particular equation. In the equations for $g$, at a particular iteration level (denoted by $\gamma+1$ ), the functions $f$ and $\theta$ are assumed to be known from the previous iteration level (denoted by $\gamma$ ). It is also assumed that all nonlinear terms in $g$ are evaluated at the previous iteration. In addition, it is also assumed that all derivative terms in $g$ which appear

$$
\begin{aligned}
& \left(1-D e f_{r}^{2}\right) g_{r+1}^{\prime \prime}+(1+M D e) f_{r} g_{r+1}^{\prime}+ \\
& 2 D e f_{r} g_{r}^{\prime} g_{r+1}-(\gamma+M) g_{r+1}=g_{r}^{2}, \\
& f^{\prime}{ }_{r+1}^{2}=g_{r+1}, \\
& \quad \frac{1}{P r} \theta_{r+1}^{\prime \prime}+f_{r+1} \theta_{r+1}^{\prime}+\left(\Omega-2 g_{r+1}\right) \theta_{r+1}
\end{aligned}
$$




$$
=-E c\left[M g_{r+1}^{2}+\left(g_{r+1}{ }^{\prime}\right)^{2}\right]=0 \text {. }
$$

The boundary conditions are the same as (19) and (20) but are now evaluated at the current iteration $(\mathrm{r}+1)$.

The equations for the algorithm (21 - 23) form a system of decoupled linear differential equations with variable coefficients which can be solved using standard numerical methods for solving differential equations. In this work, we use the Chebyshev spectral collocation method. It is for this reason that the solution method is referred to as the spectral relaxation method. For brevity, we omit the details of the spectral methods, and refer interested readers to (Canuto 1988; Fornberg 1996; Trefethen 2000). Spectral methods are well known to give remarkably good accuracy with relatively few grid points compared to other methods such as finite differences and finite element methods. This is particularly true for problems with smooth solutions.

To allow for the numerical implementation of the pseudo-spectral method, the physical region $[0, \infty)$ is truncated to $\left[0, \eta_{\infty}\right]$ where $\eta_{\infty}$ is chosen to be sufficiently large. The truncated region is further transformed to the space $[-1,1]$ using the transformation $\xi=\frac{2}{\eta_{\infty}} \eta-1$.

The spatial domain is then discretized using the popular Gauss-Lobatto collocation points (see for example Canuto 1988; Trefethen 2000) to define the nodes in [$1,1]$ as

$\xi_{j}=\cos \left(\frac{\pi j}{N}\right), \quad j=0,1, \ldots, N$

Where $(N+1)$ is the number of collocation points. The basic idea behind the Chebyshev spectral collocation method is the introduction of a differentiation matrix $D$ which maps a vector of the function values $F=$ $\left[f\left(\xi_{0}\right), \ldots, f\left(\xi_{N}\right)\right]^{T}$ at the collocation points to a vector $F^{\prime}$ defined as

$F^{\prime}=\sum_{k=0}^{N} D_{j k} f\left(\xi_{k}\right)=D F$.

In general, the derivative of order $p$ for the function $f(\eta)$ can be expressed by

$f^{(p)}(\eta)=D^{p} F$.

where $D=2 D / \eta_{\infty}$. The matrix $D$ is of size $(N+$ $1) \times(N+1)$ and its entries are defined (Canuto 1988; Trefethen 2000) as

$D_{j k}=\frac{c_{j}}{c_{k}} \frac{(-1)^{j+k}}{\xi_{j}-\xi_{k}} j \neq k ; j, k=0,1, \ldots, N$,

$D_{k k}=-\frac{\xi_{k}}{2\left(1-\xi_{k}^{2}\right)} k=1,2, \ldots, N-1$,

$D_{00}=\frac{2 N^{2}+1}{6}=-D_{N N}$,

With

$c_{k}=\left\{\begin{array}{lr}2 & k=0, N \\ 1 & -1 \leq k \leq N-1 .\end{array}\right.$
Thus, applying the spectral method, with derivative matrices on the iteration $(21-23)$ and the corresponding boundary conditions yield

$$
\begin{aligned}
& A_{1} G_{r+1}=B_{1}, g_{r+1}\left(\xi_{N}\right)=1, g_{r+1}\left(\xi_{0}\right)=0 \\
& A_{2} F_{r+1}=B_{2}, \quad f_{r+1}\left(\xi_{N}\right)=0 \\
& A_{3} \Theta_{r+1}=B_{3}, \theta_{r+1}\left(\xi_{N}\right)=1, \theta_{r+1}\left(\xi_{0}\right)=0
\end{aligned}
$$

Where

$$
\begin{array}{r}
A_{1}=D^{2}+(1+M D e) \operatorname{diag}\left[F_{r}\right] D \\
-D e d i a g\left[F_{r}^{2}\right] D^{2} \\
+2 \text { Dediag }\left[F_{r} G_{r}^{\prime}\right]-(M+\gamma) I, \quad B_{1}=G_{r}^{2},
\end{array}
$$

$A_{2}=D, \quad B_{2}=G_{r+1}$.

$A_{3}=D^{2}-2 \operatorname{Prdiag}\left(G_{r}\right) D+\operatorname{Prdiag}\left(F_{r}\right)+\Omega I$,

$B_{3}=-\operatorname{PrEc}\left[M G_{r}^{2}+G_{r^{\prime}}^{2}\right]$,

with $I$ being an identity matrix of size $(N+1) \times$ $(N+1), G, F$ and are the values of functions $f$, $g$ and $\theta$ respectively, when evaluated at the collocation points. Equations (32 - 34) constitute the SRM iteration scheme. The initial approximations required to start the iteration process are $g_{0}(\eta)=$ $e^{-\eta}, f_{0}=1-e^{-\eta}$ and $\theta_{0}=e^{-\eta}$ which are convenient functions that satisfy all the boundary conditions. Starting from $f_{0}, g_{0}, \theta_{0}$, the SRM scheme is implemented repeatedly for $r=1,2, \ldots$, until the difference between successive solutions is less than a given tolerance level. In this work, convergence of the SRM scheme was defined in terms of the infinity norm as

$$
\begin{gathered}
E_{r}=\operatorname{Max}\left(\left\|f_{r+1}-f_{r}\right\|_{\infty},\left\|g_{r+1}-g_{r}\right\|_{\infty}\right. \\
\left.\left\|\theta_{r+1}-\theta_{r}\right\|_{\infty}\right)
\end{gathered}
$$

If the iteration scheme converges, the error $E_{r}$ is expected to decrease with an increase in the number of iterations. Accuracy of the scheme was established by increasing the number of collocation points $N$ until the solutions are consistent and further increase does not change the value of the solutions.

The convergence of the SRM can be improved by employing successive over-relaxation (SOR) like methods which are widely used to accelerate the convergence rates of the Gauss-Seidel method in the solution of linear systems of equation. If the SRM scheme for obtaining the function $X$ at the $(r+1)$ th iteration is

$A X_{r+1}=B$,

then the modified version of the SRM is defined as

$A X_{r+1}=(1-\omega) A X_{r}+\omega B$

where $A, B$ are matrices and $\omega$ is the convergence controlling relaxation parameter. It must be noted that 
when $\omega=1$ equation (37) reduces to the original SRM method. For some parameters of $\omega$ near 1 , it will be demonstrated in the next section that the convergence of the SRM is improved.

\section{Results AND Discussion}

The magnetohydrodynamic flow and heat transfer in a Maxwell fluid over a stretching sheet in a porous medium has been solved numerically using a spectral relaxation method (SRM). Numeric expressions of the velocity and temperature have been used to compute the entropy generation. The results are displayed in this section showing a comparison between the SRM

Table 1 Comparison of SRM solutions for $-f^{\prime \prime}(0)$ against those of the bvp4c for different values of $M, D e$ and $\gamma$ when $N=60$ obtained at different values of $\eta_{\infty}$.

\begin{tabular}{|c|c|c|c|c|c|c|c|}
\hline \multicolumn{2}{|c|}{} & \multicolumn{2}{c|}{ SRM } & \multicolumn{2}{c|}{ With SOR } & & \\
\hline$M$ & $D e$ & $\gamma$ & iter. & $\omega$ & iter. & $-f^{\prime \prime}(0)$ & bvp4c \\
\hline 1 & 1 & 1 & 18 & 0.90 & 12 & 1.96971626 & 1.96971626 \\
\hline 2 & 1 & 1 & 17 & 0.90 & 12 & 2.28778217 & 2.28778217 \\
\hline 3 & 1 & 1 & 16 & 0.95 & 10 & 2.56666187 & 2.56666187 \\
\hline 1 & 0.5 & 1 & 17 & 0.90 & 12 & 1.85307105 & 1.85307105 \\
\hline 1 & 1 & 1 & 18 & 0.90 & 12 & 1.96971626 & 1.96971626 \\
\hline 1 & 2 & 1 & 22 & 0.95 & 17 & 2.18931579 & 2.18931579 \\
\hline 1 & 1 & 0.5 & 21 & 0.90 & 12 & 1.84034071 & 1.84034071 \\
\hline 1 & 1 & 1 & 18 & 0.90 & 12 & 1.96971626 & 1.96971626 \\
\hline 1 & 1 & 2 & 16 & 0.95 & 11 & 2.20681301 & 2.20681301 \\
\hline
\end{tabular}

Table 2 Comparison of SRM solutions for $-\theta^{\prime}(0)$ against those of the bvp4c for different values of $P r, E c$ and $M$ when $N=60$ obtained at different values of $\eta_{\infty}$.

\begin{tabular}{|c|c|c|c|c|c|c|c|}
\hline \multicolumn{2}{|l}{} & SRM & \multicolumn{2}{c|}{ With SOR } & & \\
\hline$P r$ & $E c$ & $M$ & iter & $\omega$ & iter & $-\theta^{\prime}(0)$ & bvp4c \\
\hline 3 & 0.2 & 1 & 18 & 0.90 & 12 & 2.09594309 & 2.09594309 \\
\hline 5 & 0.2 & 1 & 18 & 0.90 & 12 & 2.72342818 & 2.72342818 \\
\hline 7 & 0.2 & 1 & 18 & 0.90 & 12 & 3.21999625 & 3.21999625 \\
\hline 5 & 0.5 & 1 & 18 & 0.90 & 12 & 1.59114632 & 1.59114632 \\
\hline 5 & 1 & 1 & 18 & 0.90 & 12 & -0.29599013 & -0.29599013 \\
\hline 5 & 1.5 & 1 & 18 & 0.90 & 12 & -2.18312657 & -2.18312657 \\
\hline 5 & 0.2 & 1 & 18 & 0.90 & 12 & 2.72342818 & 2.72342818 \\
\hline 5 & 0.2 & 2 & 17 & 0.90 & 12 & 2.38301961 & 2.38301961 \\
\hline 5 & 0.2 & 3 & 16 & 0.95 & 10 & 2.08191143 & 2.08191143 \\
\hline
\end{tabular}

A plot of the error of the norm, as defined in equation (35), against the number of iterations has been used in Figure 2 to illustrate how the SRM error reduces with increased number of iterations. The plot has been generated for different values of the magnetic parameter. A steep decrease of the error is being observed for all values of $M$ The decrease in the error is an indication that the method is convergent and gives stable solutions. The convergence rate is taken to be the number of iterations the solution takes to reach a specified accuracy level. For example, in the figure it shows that when $M=4$ the solution will converge after fourteen iterations for the given accuracy level compared to sixteen when $M=2$ solutions with those generated using the bvp $4 \mathrm{c}$ and effects of selected flow parameters on the velocity, temperature and entropy generation number. The comparison was made to validate the accuracy of the SRM solutions. The bvp4c is an in-built MATLAB solver for boundary value problems based on the fourth order Runge-Kutta schemes. The tolerance level for both methods was set to be $10^{-10}$. The figures were generated using

$N=100$ and $\eta_{\infty}=20$ unless specified. 
generated at selected values of the magnetic parameter, the Deborah number and the porosity parameter. The Table also displays the advantage of accelerating the convergence rate. An improvement in the convergence rate is observed for all cases. The effect of the magnetic parameter, Deborah number and porosity parameter is to increase the shear stress. A very good agreement between the two solutions for up to eight decimal places is being observed.

The heat transfer rate $-\theta^{\prime}(0)$ for selected values of the Prandtl number, Eckert number and the magnetic parameter is shown in Table 2. The comparison between the SRM and bvp4c results shows a very good agreement for up to eight decimal places. Heat transfer rate increases with increase in $\mathrm{Pr}$ while decreases with increase in $E c$ and $M$. In the Table the advantage of accelerating the convergence is again shown. The convergence rates improve for all cases with relaxation.

The variation of the transverse velocity $f(\eta)$ for different values of the magnetic parameter $M$ is shown in Fig. 3. For any $M, f(\eta)$ increases with $\eta$ asymptotically. Applying a magnetic field decreases the momentum boundary layer thickness and increase the power required to stretch the sheet. This in turn decreases $f(\eta)$ with increase in the magnetic parameter. While $f(\eta)$ increases asymptotically with $\eta$, the longitudinal velocity, $f^{\prime}(\eta)$ is seen to decrease with

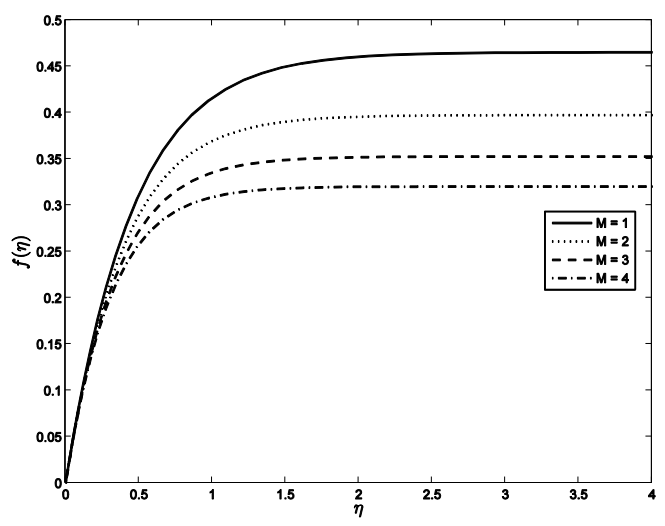

Fig. 3.Effect of the magnetic parameter on the transverse velocity $(D e=1$ and $\gamma=1)$.

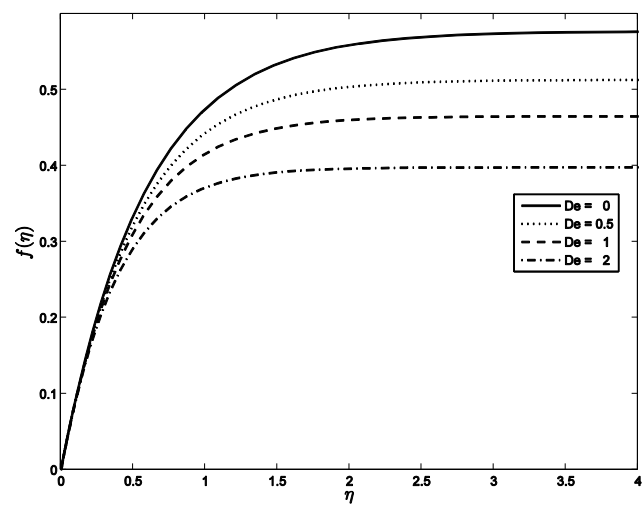

Fig. 5.Effect of the Deborah number on the transverse velocity $(M=1$ and $\gamma=1)$.

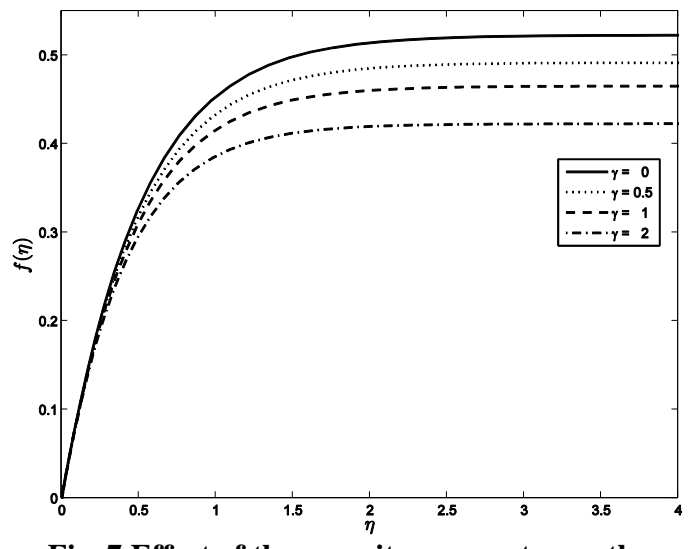

Fig. 7.Effect of the porosity parameter on the transverse velocity $(M=1$ and $D e=1)$.

$\eta$ in Fig. 4. For the same justification as in Fig. 3 the longitudinal velocity is seen to decrease with increase in the magnetic parameter. Application of the transverse magnetic field produces a drag force known as Lorentz force thereby decreasing the magnitude of the flow velocity.

The effect of the Deborah number is shown to decrease the transverse velocity $f(\eta)$ of the flow in Fig. 5. In Fig. 6 the longitudinal velocity $f^{\prime}(\eta)$ is seen to also decrease with increase in the Deborah number.

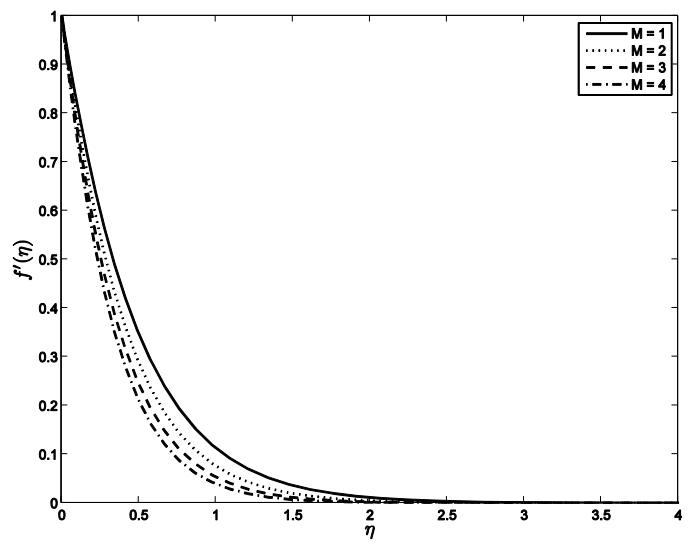

Fig. 4.Effect of the magnetic parameter on the longitudinal velocity $(D e=1$ and $\gamma=1)$.

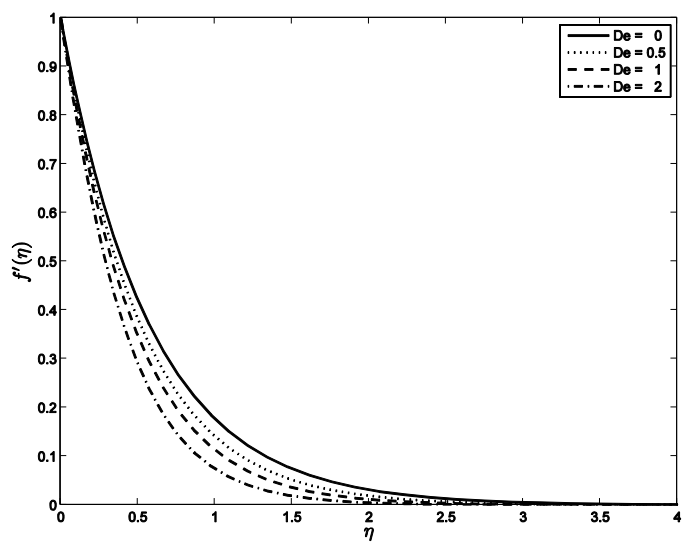

Fig. 6. Effect of the Deborah number on the longitudinal velocity $(M=1$ and $\gamma=1)$. 


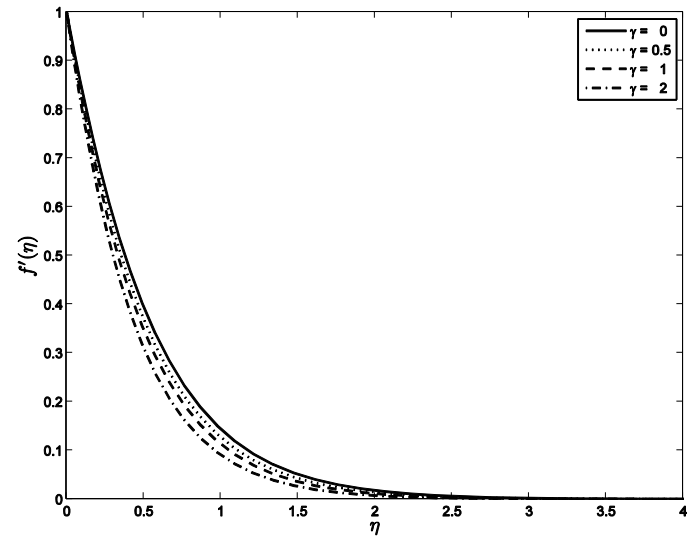

Fig. 8. Effect of the porosity parameter on the longitudinal velocity $(M=1$ and $D e=1)$.

In Fig. 7 is the effect of the porosity parameter on the transverse velocity of the flow. Increasing the porosity of the surface tends to decrease the transverse velocity of the flow. The longitudinal velocity $f^{\prime}(\eta)$ is also seen to decrease with increase in the porosity parameter in Fig. 8.

The temperature profiles for different values of the magnetic parameter are shown in Fig. 9. Thermal boundary layer increases in the presence of a magnetic field, thus increasing the magnetic parameter results in

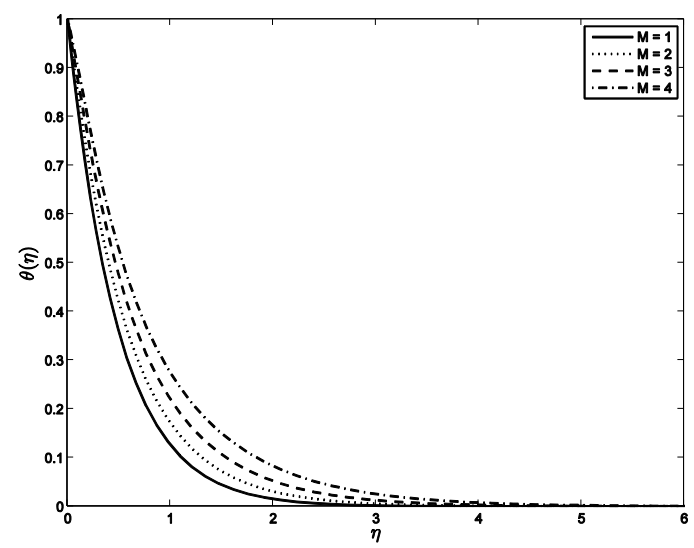

Fig. 9.Effect of the magnetic parameter on the temperature $(E c=0.2, \operatorname{Pr}=3, \Lambda=0.1)$.

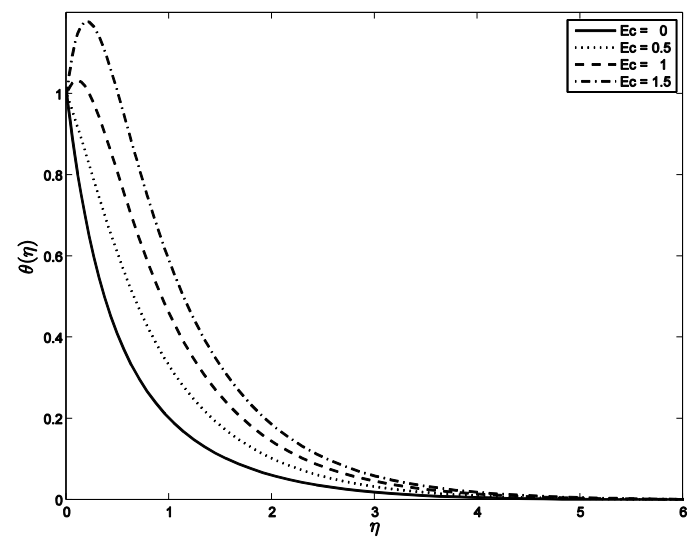

Fig. 11.Effect of the Eckert number on the temperature $(\operatorname{Pr}=3, M=1, \Lambda=0.1)$. an increase of the temperature. The presence of a transverse magnetic field slows down the fluid flow thereby heating up the fluid. This in turn reduces the heat transfer from the wall which then leads to the thickening of the thermal boundary layer.

The effect of the Prandtl number on the temperature is shown in Fig. 10. The temperature decreases with an increase in Prandtl number which implies that the thermal boundary layer becomes thinner with large Prandtl number.

Figure 11 shows the effect of the Eckert number on the temperature profile. High $E c$ numbers implies highheat levels produced by friction hence increased temperatures on the system. Temperature profiles as functions of $\eta$ for various values of the heat source parameter are shown in Fig. 12. The temperature increases with the heat source parameter for a fixed value of $\eta$. This happens because increasing the heat source/sink parameter means increased heat generated inside the boundary layer leading to higher temperature profile.

The effect of the magnetic parameter on the entropy generation number is shown on Fig. 13. The entropy generation number increases with the magnetic parameter. This happens because the magnetic field supports entropy in the fluid. Physically the presence of

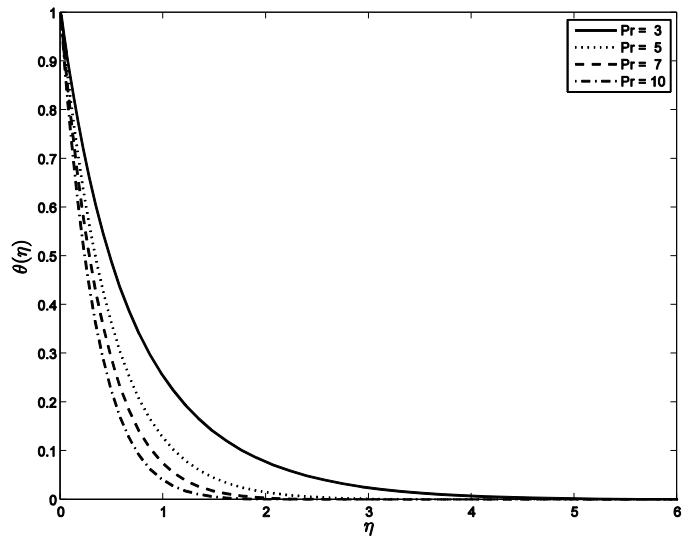

Fig. 10. Effect of the Prandtl number on the temperature $(E c=0.2, M=1, \Lambda=0.1)$.

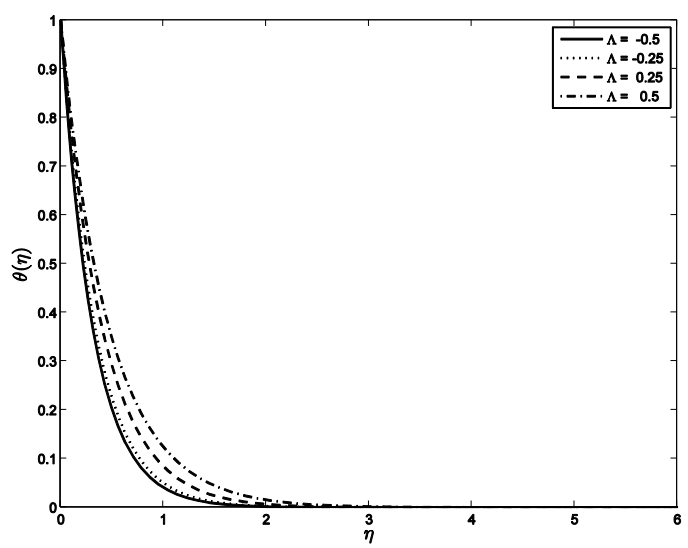

Fig. 12. Effect of the heat source on the temperature $(P r=3, M=1, E c=0.2)$. 
the magnetic field creates more entropy generation in the fluid as the fluid as fluid flow velocity is reduced. Figure 14 presents the effect of the Prandtl number $P r$ on the entropy generation number. The entropy generation number is higher for lower Prandtl number. Increasing values of the Prandtl number reduces the fluid temperature as highlighted in Figure 10. This sharp decrease of the temperature profiles leads to sharp decrease in the entropy generation in the flow system. For a given value of $\eta$, the entropy generation number increases with increase in the Reynolds number. This is shown in Fig. 15. At high Reynolds numbers fluid friction and heat transfer take place in the boundary layer increasing the contribution of the entropy generation number as the fluid becomes more viscous. The effect of the dimensionless group parameter $\mathrm{Br} \Omega^{-1}$ on the entropy generation number $N_{S}$ is shown in Fig. 16. The relative importance of the viscous effect on the flow is determined by this parameter. In the figure, for a given value of $\eta$ the entropy generation number is higher for higher dimensionless group. This is caused by the fact that for higher dimensionless group, the entropy generation numbers due to the fluid friction are increased.

\section{CONCLUSION}

A spectral relaxation method (SRM) has been successfully used to solve numerically the

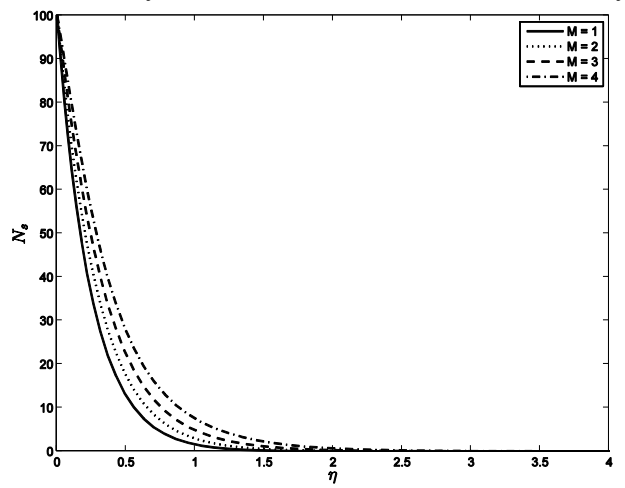

Fig. 13.Effect of the magnetic parameter on the entropy generation number $(\mathrm{De}=1, \gamma=1, \operatorname{Pr}=$ 5, $\Lambda=0.1, E c=0.2, R e_{l}=0.1, X=0.2$, $B r \Omega^{-1}=0.1, H a=0.1$ ).

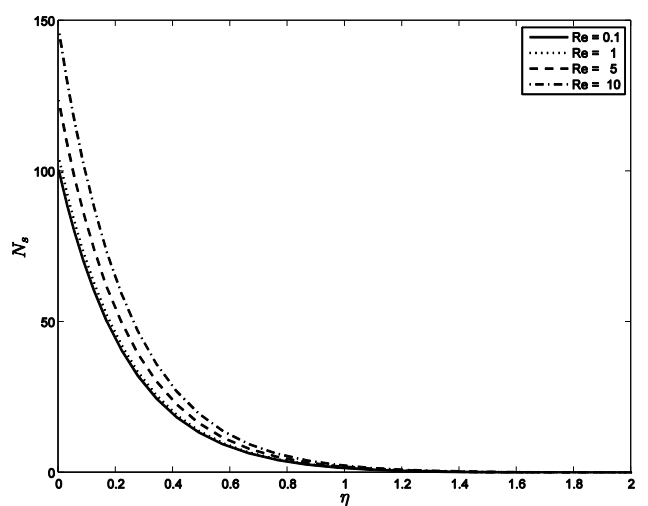

Fig. 15.Effect of the Reynolds number on the entropy generation number $(D e=1, \gamma=1$,

$P r=5, \Lambda=0.1, E c=0.2, M=1, X=0.2$, $\left.B r \Omega^{-1}=0.1, H a=0.1\right)$. magnetohydrodynamic flow and heat transfer in a Maxwell fluid over a stretching surface in a porous medium. The entropy generation number was computed using numeric expressions of the velocity and temperature. Effects of the magnetic parameter, Prandtl number, Reynolds number and dimensionless group parameter on the entropy generation number have been investigated and discussed. Discussed also was the effect of the Deborah number, the magnetic parameter and the porosity parameter on the velocity of the flow and that of the Prandtl number, Eckert number, magnetic parameter and heat source/sink parameter on the temperature of the flow. It was found that velocities depend strongly on the magnetic and the viscoelastic parameters while the temperature varied significantly with the Prandlt number, the magnetic parameter, the Eckert number and the heat source parameter. Entropy

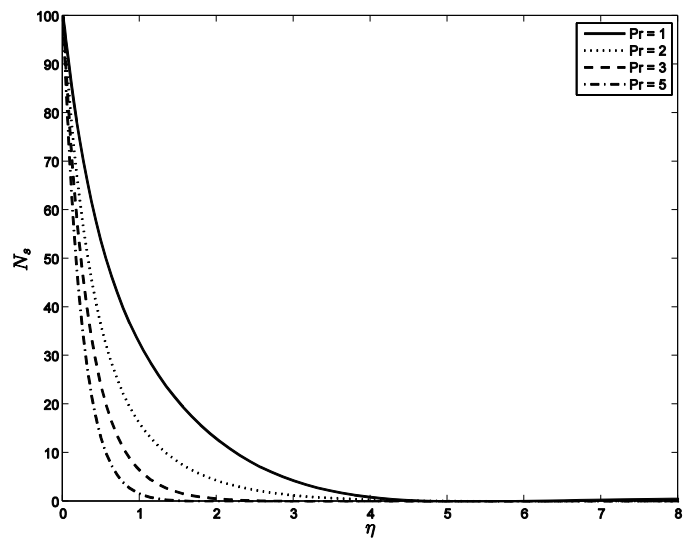

Fig. 14.Effect of the Prandtl number on the entropy generation number $(D e=1, \gamma=1, M=1, \Lambda=$ $0.1, E c=0.2, R e_{l}=0.1, X=0.2$, $\left.B r \Omega^{-1}=0.1, H a=0.1\right)$.

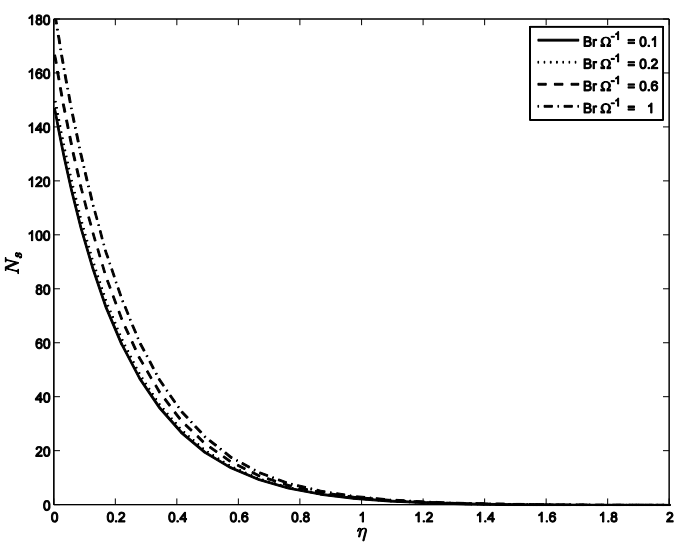

Fig. 16.Effect of the dimensionless group on the entropy generation number $(D e=1, \gamma=1, P r=$

5, $\Lambda=0.1, E c=0.2, M=1, X=0.2$, $R e_{l}=10, H a=0.1$ ).

generation increased with the Reynolds number, the magnetic parameter and the dimensionless group parameter while decreased for higher Prandtl numbers. The spectral relaxation method was found to be accurate and rapidly convergent to the numerical results. 


\section{ACKNOWLEDGEMENTS}

The authors wish to acknowledge financial support from the University of Venda, University of KwaZulu-

\section{REFERENCES}

Abel,M. S., J.V.Tawade, and M. M. "Nandeppanavar (2012). MHD flow and heat transfer for the upperconvected Maxwell fluid over a stretching sheet,'Meccanica, 47, pp 385-393.

Abel, M.S, J. V. Tawade, and J. N. Shinde (2012).“The effects of MHD flow and heat transfer for the UCM Fluid over a stretching surface in presence of thermal radiation,"Advances in Mathematical Physics, vol 2012, Article ID 702681, 21 pages doi:10.1155/2012/702681.

Ahmed, S., and K. Kalita (2013)."Analytical and Numerical Study for MHD Radiating Flow over an Infinite Vertical Surface Bounded by a Porous Medium in Presence of Chemical Reaction,"Journal of Applied Fluid Mechanics. 6(4), 597-607.

Bataller, R. C. (2011).“Magnetohydrodynamic flow and heat transfer of an upper-convected Maxwell fluid due to a stretching sheet,"FDMP, 7(2), 153-173.

Bejan, A. (1979).“A study of entropy generation in fundamental convective heat transfer," J. Heat Transfer, 101, 718-725.

Bejan, A. (1982)."Second-law analysis in heat transfer and thermal design,"Adv. Heat Transfer, 15, 1-58.

Butt, A. S., and A. Ali (2013).“Effects of magnetic field on entropy generation in flow and heat transfer due to a radially stretching surface,"Chin. Phys. Lett. 30(2), 024701.

Butt, A. S., S.Munawar, A. Ali and A. Mehmood (2012)."Entropy generation in hydrodynamic slip flow over a vertical plate with convective boundary,"Journal of Mechanical Science and Technology, 26 (9), 2977-2984.

Canuto, C., Hussaini, M. Y., A. Quarteroni, and T. A. Zang (1988).Spectral Methods in Fluid Dynamics, Springer-Verlag, Berlin.

Chen, C. O-K., Y. -T. Yang and K. -H. Chang (2011)."The effect of thermal radiation on entropy generation due to micro-polar fluid flow along a wavy surface,"Entropy, 13, 1595-1610.

Eegunjobi, A. S., and O. D. Makinde (2012). "Combined effect of buoyancy force and navier slip on entropy generation in a vertical porous channel," Entropy, 14, 1028-1044.
Natal and the National Research Foundation of South Africa (Grant No: 85596).

Fornberg, B (1996). A Practical Guide to Pseudospectral Methods, Cambridge Univ. Press, New York.

Hayat, T., M. Farooq, Z. Iqbal and A. Alsaedi (2012)."Mixed convection Falkner-Skan flow of a Maxwell fluid,"Journal of Heat Transfer, 134, 114504.

Hayat T., R. Sajjad, Z. Abbas, M. Sajid and A. A. "Hendi (2011). Radiation effects on MHD flow of Maxwell fluid in a channel with porous medium,"International Journal of Heat and Mass Transfer, 54, 854-862.

Hassan, M., R. Sadri, G. Ahmadi, M. B. Dahari, S. N. Kazi, M. R. Safaei and E. Sadeghinezhad (2013)."Numerical study of entropy generation in a flowing nanofluid used in micro- and minichannels,"Entropy, 15, 144-155.

Karamallah, A. A., W. S.,Mohammad, and W. H. Khalil (2011)."Numerical study of entropy generation in a vertical square channel packed with saturated porous media,"Eng. \& Tech. Journal, 29(9), 1721-1736.

Kumar, H.,(2013)."Radiative Heat Transfer with MHD Free Convection Flow over a Stretching Porous Sheet in Presence of Heat Source Subjected to Power Law Heat Flux,"Journal of Applied Fluid Mechanics, 6 (4), 563-569.

Mahian, O., S. Mahmud and S. Z. Heris (2012)."Effect of uncertainties in physical properties on entropy generation between two rotating cylinders with nanofluids,"Journal of Heat Transfer, 134, 101704,

Makinde, O. D., A. Aziz, (2011). “ Analysis of entropy generation and thermal stability in a long hollow cylinder with asymmetry convective cooling,"Heat Mass Transfer, 47, 1407-1415.

Makinde, O. D., and A. W. Gbolagade, (2005)“Second law analysis of incompressible viscous flow through an inclined channel with isothermal walls,"Rom. Journ. Phys., vol. 50, nos. 9-10, pp. 923-930, 2005.

Makinde O. D., and E. Osalusi, (2005).“Second law analysis of laminar flow in a channel filled with saturated porous media,"Entropy, 7(2), 148-160,

Mamaloukas Ch., M. Subhas Abel, J. V. Tawade, and U. S. Mahabaleswar, (2011)."On effects of a transverce magnetic field on an UCM fluid over a stretching sheet,"International Journal of Pure and Applied Mathematics, 66 (1), 1-9. 
Motsa, S. S., Hayat, T., and O. M. Aldossary,(2012).“MHD flow of upper-convected Maxwell fluid over porous stretching sheet using successive Taylor series linearization method,"Appl. Math. Mech. -Engl. Ed., 33(8), 975-990.

Motsa S. S., and Z. G. Makukula, (2013).“On spectral relaxation method approach for steady von Kármán flow of a Reiner-Rivlin fluid with Joule heating and viscous dissipation,"Central European Journal of Physics, 11 (3), 363-374.

Mukhopadhyay, S. (2012)."Heat transfer analysis of the unsteady flow of a Maxwell fluid over a stretching surface in the presence of a heat source/sink,"Chin. Phys. Lett., 29 (5), 054703.

Mukhopadhyay S. and R. S. R. Gorla, (2012)."Unsteady MHD boundary layer flow of an upper convected Maxwell fluid past a stretching sheet with first order constructive/destructive chemical reaction,"Journal of Naval Architecture and Marine Engineering, 9,123-133.

Muthucumaraswamy R, Dhanasekar N. and Prasad, G. E., (2013). "Rotation Effects on Unsteady Flow past an Accelerated Isothermal Vertical Plate with Variable Mass Transfer in the Presence of Chemical Reaction of First Order,"Journal of Applied Fluid Mechanics, 6(4), 485-490.

Noor, N. F. M., (2012). “Analysis for MHD flow of a Maxwell fluid past a vertical stretching sheet in the presence of thermophoresis and chemical reaction," World Academy of Science, Engineering and Technology, 64, 1019-1023.

Rashidi, M. M. and FreidooniMehr, N.(2012)."Effects of velocity slip and temperature jump on the entropy generation in magnetohydrodynamic flow over a porous rotating disk,"Journal of Mechanical Engineering, vol. 1, no. 3, pp. 4-14.

Shateyi, S. and Makinde, O. D., (2013).“Hydromagnetic Stagnation-Point Flow towards a Radially Stretching Convectively Heated Disk,"Mathematical Problems in Engineering, Volume 2013, Article ID 616947, 8 pages http://dx.doi.org/10.1155/2013/616947.

Shuja, S. Z., Yilbas, B. S., and A. Jamal, (2008)."Entropy generation in flow field subjected to a porous block in a vertical channel,"Transp. Porous Med., 72, 179-197.

Torabi, M., and A. Aziz, (2012)."Entropy generation in a hollow cylinder with temperature dependent thermal conductivity and internal heat generation with convective-radiative surface cooling,"International Communications in Heat and Mass Transfer, 39, 1487-1495.

Tshehla, M. S., Makinde, O. D., and G. E. Okecha, (2010).Heat transfer and entropy generation in a pipe flow with temperature dependent viscosity and convective cooling, Scientific Research and Essays, 5 (23), 3730-3741.

Tshehla, M. S., and O. D. Makinde, (2011).“Analysis of entropy generation in a variable viscosity fluid flow between two concentric pipes with a convective cooling at the surface,"International Journal of the Physical Sciences, 6(25), 60536060.

Trefethen, L. N, (2000)Spectral Methods in MATLAB, SIAM, 